\title{
Newtonian Relaxation
}

The model simulates boundary layer processes that affect cloud microphysics on timescales of seconds to hours. To simulate the effect of processes that occur on longer time scales and other processes that are not included in the model, such as horizontal advection, the model uses Newtonian relaxation (nudging) techniques. The nudging time scale of temperature was set to the model time step (900 s) so that it was completely constrained by observations. This was done so that water cycle processes in the model would be simulated at the specified temperatures.

Between the lowest initial condition and the upper boundary conditions, Newtonian relaxation is used with varying time scales for momentum and moisture. Since advection is assumed to be less important than turbulence and cloud microphysics at these altitudes, the momentum time scale was set to three hours. This allowed us to maintain realistic wind profiles but allowed some of the noisier wind data to be smoothed out (short-term eddies may have been included in the observational data). The model physics could thus account for the impacts of turbulent mixing in the boundary layer on horizontal winds, but provides a constraint to keep the model from diverging too far from realistic values.

Above the boundary layer height, all variables were also nudged at every time step since the model is only representative where thermodynamic conditions are determined primarily by large-scale processes. This provides an upper boundary condition in the model, by providing a constant flux of moisture. The moisture flux in the top portion of the cloud, where we examined the model output, was found to be mostly insensitive to the momentum flux at a time scale of 3 hours.

Moisture variables were completely dictated by the model physics below the upper boundary condition. This allowed thermodynamic equilibrium to be reached at the end of our model run. LWC, the slowest variable to equilibrate, came close to equilibrium after roughly 100 hours. 